\title{
Tortuosity of Battery Electrodes: Validation of Impedance-Derived Values and Critical Comparison with 3D Tomography
}

\author{
Johannes Landesfeind, $\oplus^{1, *, z}$ Martin Ebner, ${ }^{2}$ Askin Eldiven, ${ }^{1}$ Vanessa Wood, $\oplus^{2, * *}$ \\ and Hubert A. Gasteiger ${ }^{1, * * *}$
}

${ }^{1}$ Chair of Technical Electrochemistry, Department of Chemistry and Catalysis Research Center,

Technical University of Munich, Munich, Germany

${ }^{2}$ Department of Information Technology and Electrical Engineering, ETH Zurich, Zurich, Switzerland

\begin{abstract}
Tortuosity values of porous battery electrodes determined using electrochemical impedance spectroscopy in symmetric cells with a non-intercalating electrolyte are typically higher than those values based on numerical analysis of 3D tomographic reconstructions. The electrochemical approach assumes that the electronic resistance in the porous coating is negligible and that the tortuosity of the porous electrode can be calculated from the ionic resistance determined by fitting a transmission line equivalent circuit model to the experimental data. In this work, we validate the assumptions behind the electrochemical approach. First, we experimentally and theoretically investigate the influence of the electronic resistance of the porous electrode on the extracted ionic resistances using a general transmission line model, and provide a convenient method to determine whether the electronic resistance is sufficiently low for the model to be correctly applied. Second, using a macroscopic setup with known tortuosity, we prove that the ionic resistance quantified by the transmission line model indeed yields the true tortuosity of a porous medium. Based on our findings, we analyze the tortuosities of porous electrodes using both X-ray tomography and electrochemical impedance spectroscopy on electrodes from the same coating and conclude that the distribution of the polymeric binder phase, which is not imaged in most tomographic experiments, is a key reason for the underestimated tortuosity values calculated from 3D reconstructions of electrode microstructures.

(c) The Author(s) 2018. Published by ECS. This is an open access article distributed under the terms of the Creative Commons Attribution Non-Commercial No Derivatives 4.0 License (CC BY-NC-ND, http://creativecommons.org/licenses/by-nc-nd/4.0/), which permits non-commercial reuse, distribution, and reproduction in any medium, provided the original work is not changed in any way and is properly cited. For permission for commercial reuse, please email: oa @electrochem.org. [DOI: 10.1149/2.0231803jes]

(cc) BY-NC-ND
\end{abstract}

Manuscript submitted December 28, 2017; revised manuscript received January 26, 2018. Published February 10, 2018.

In commercially relevant lithium ion battery cells operating at high currents or low temperatures and/or cells with thick and low porosity electrodes (i.e., electrodes with high areal capacity and high volumetric energy density), the ionic transport in the electrolyte throughout the thickness of the porous electrode becomes limiting, leading to the buildup of excessive electrolyte concentration gradients across the thickness of the electrode. Concentration gradients not only lead to increased overpotentials and thus lower accessible capacities, but also play an important role in battery aging caused by lithium plating reactions at the graphite anode/separator interface. ${ }^{1}$ Along with the intrinsic transport parameters of the liquid electrolyte, the morphological properties of a porous electrode, quantified by the parameters porosity and tortuosity, are key to understanding the buildup of concentration gradients across the electrode thickness and the resulting performance limitations of porous electrodes.

In the battery community, there are currently two commonly used approaches to obtain values for the tortuosity of porous electrodes; however, they yield different results. One is based on numerical diffusion simulations on $3 \mathrm{D}$ reconstructions of the electrode obtained using X-ray (XTM) or focused ion beam scanning electron microscopy (FIB SEM) tomography. ${ }^{2,3}$ The other approach is based on electrochemical impedance spectroscopy (EIS) measurements of the electrodes in a symmetric cell configuration. ${ }^{4-6}$ Reported tortuosity values from the EIS method consistently yield larger tortuosity values compared to the ones reported from the numerical 3D approach. ${ }^{5}$

To obtain tortuosities of porous electrodes from impedance spectra of symmetric cells, it is assumed that a) the electronic resistance of the solid phase of the porous electrode is negligible, and, b) that the ionic resistance determined from fitting a transmission line model equivalent circuit to the impedance data represents the correct electrode tortuosity values. In this work, we investigate the validity of these two assumptions and then proceed to analyze the origin of the discrepancy between the tortuosity values derived from $3 \mathrm{D}$ reconstructions based on X-ray tomography compared to EIS-derived values.

\footnotetext{
*Electrochemical Society Student Member.

***Electrochemical Society Member.

***Electrochemical Society Fellow.

${ }^{\mathrm{z}}$ E-mail: j.landesfeind@tum.de
}

Validation of the first assumption is important because the simplified transmission model used to analyze the EIS assumes electronic resistances are negligible. While for some electrodes, such as graphite, it is reasonable to assume good electronic conductivity, for cathodes with active materials having low electronic conductivity (e.g., LNMO $\left.\left(\mathrm{LiNi}_{0.5} \mathrm{Mn}_{1.5} \mathrm{O}_{4}\right)\right)$, it is unclear whether the electronic resistance contribution within the electrode is negligible. We therefore use a general transmission line model to study the influence of the electronic conductivity of an electrode on the measured ionic conductivity of the electrolyte within the electrode pores and propose how to approach measuring the tortuosity of electrodes with unknown electronic conductivity via EIS.

To validate the second assumption that one can link the measured ionic resistance to the tortuosity, we apply the EIS method to a system of known tortuosity. Specifically, we construct a macroscopic model of an electrode, consisting of conducting spheres which are densely packed into a cylindrical tube and separated mid-way by a porous separator. We demonstrate that the EIS-derived tortuosity value in the macroscopic setup is indeed identical to the value predicted by the Bruggeman equation.

Finally, to resolve the origin of the observed discrepancy between the tortuosity values derived from 3D reconstructions based on $\mathrm{X}$-ray tomography compared to EIS-derived values, both methods are applied to identical electrodes.

\section{Experimental}

Composite electrodes and cells used in this work were prepared and measured as described previously unless stated otherwise below. ${ }^{5}$ To show the influence of the binder (Figure 6) a slurry of active material (T311, SGL Carbon, $3.0 \mathrm{~m}^{2} / \mathrm{g}$, D50 $19 \mu \mathrm{m}$ ), PVDF binder (Kureha KF 1100) and NMP (Sigma Aldrich, anhydrous, 99.5\%) was prepared in a planetary mixer (Thinky ARV-310) and doctor-blade coated on a copper current collector foil (MTI, $9 \mu \mathrm{m}$ ). All components were simultaneously loaded and mixed at $2000 \mathrm{rpm}$ for $5 \mathrm{~min}$. A 1:1 (by weight) mixture of ethylene carbonate (EC, Sigma Aldrich, anhydrous, 99\%) and diethyl carbonate (DEC, Sigma Aldrich, anhydrous, $>99 \%$ ), was used as a solvent for the self-prepared electrolytes containing tetrabutylammonium perchlorate $\left(\mathrm{TBAClO}_{4}\right.$, Sigma Aldrich, 
$\geq 99.0 \%$ ) salt. These graphite electrodes had a porosity of $\varepsilon=48 \%$, a thickness of $t=90 \mu \mathrm{m}$, and an areal capacity of $\sim 3 \mathrm{mAh} / \mathrm{cm}^{2}$ $\left(8.6 \mathrm{mg} / \mathrm{cm}^{2}\right)$. Graphite, NCA, and NMC $\left(\mathrm{Li}_{1} \mathrm{Ni}_{0.33} \mathrm{Mn}_{0.33} \mathrm{Co}_{0.33} \mathrm{O}_{2}\right)$ electrodes for the comparison of EIS and XTM tortuosities were obtained from Custom Cells (specifications in Table II).

A turn-key conductivity sensor (LF 1100+, SI Analytics, with custom made ground glass fitting) with a built-in temperature sensor was used to measure the conductivity of the used self-mixed electrolytes at $25^{\circ} \mathrm{C}$ (Sigma Aldrich). Symmetrical Swagelok type T-cells (spring-compressed to $\approx 1$ bar) were built outside the glove box using two porous glass fiber separators (binder free glass microfiber 691 , thickness $200 \mu \mathrm{m},>90 \%$ porosity, VWR), then transferred into a temperature controlled climate chamber $\left(25^{\circ} \mathrm{C}\right.$, Binder $)$, and the impedance spectra were recorded around $\mathrm{OCV}$ after a resting period of at least $12 \mathrm{~h}$ in a frequency range of $200 \mathrm{kHz}$ to $0.1 \mathrm{~Hz}$ with a 20 $\mathrm{mV}$ perturbation.

\section{Effect of the Electronic Resistance in a Porous Electrode}

First, we evaluate under which conditions the electronic resistance contributions in a porous electrode can be neglected when determining the electrode tortuosity from EIS, which is the assumption behind the use of the simplified transmission line model. ${ }^{5}$

To evaluate whether this assumption is valid for porous electrodes, we consider the general formulation of the transmission line model, ${ }^{7}$ where the electronic resistance in the solid phase and the ionic resistance in the liquid electrolyte phase are considered, whereby both contributions impact the characteristic $45^{\circ}$ mid-frequency section of the impedance in the associated Nyquist plot. Adopting the nomenclature of our previous work, the general transmission line model for a porous electrode $\left(Z_{\mathrm{El}}\right)$ can be described by ${ }^{7}$

$$
Z_{\mathrm{El} .}=Z_{\|}+Z^{*} \frac{1+2 \cdot p \cdot s\left[\sqrt{1-\tanh (\nu)^{2}}-1\right]}{\tanh (\nu)}
$$

with

$$
\begin{gathered}
Z_{\|}=\frac{Z_{1} \cdot Z_{2}}{Z_{1}+Z_{2}} \\
Z^{*}=\sqrt{\left(Z_{1}+Z_{2}\right) \cdot Z_{\mathrm{S}}} \\
p=\frac{Z_{2}}{Z_{1}+Z_{2}} \\
s=\frac{Z_{1}}{Z_{1}+Z_{2}} \\
\nu=\sqrt{\frac{Z_{1}+Z_{2}}{Z_{\mathrm{S}}}}
\end{gathered}
$$

Here, $Z_{1}, Z_{2}$, and $Z_{\mathrm{S}}$ are the impedances of the electron conducting solid phase of the electrode $\left(Z_{1} \equiv R_{\mathrm{El}}\right)$, of the ionically conducting electrolyte phase within the pores of the electrode $\left(Z_{2} \equiv R_{\text {Ion }}\right)$, and of the solid/electrolyte surface impedance within the electrode $Z_{\mathrm{S}}$. In general, the surface impedance element $Z_{\mathrm{S}}$ is described by an $R / Q$ element, composed of the constant phase capacitance $\left(Q_{\mathrm{S}}\right)$ and the charge transfer resistance $\left(R_{\mathrm{CT}}\right)$

$$
Z_{\mathrm{S}}=\frac{R_{\mathrm{CT}}}{R_{\mathrm{CT}} \cdot(\mathrm{i} \omega)^{\gamma} Q_{\mathrm{S}}+1}
$$

with the angular frequency $\omega$ and the constant phase exponent $\gamma$. If no charge transfer reactions occur $\left(R_{\mathrm{CT}} \rightarrow \infty\right)$, only the capacitive coupling remains and Eq. 7 becomes (compare also Ref. 5)

$$
Z_{\mathrm{S}}=\frac{1}{(i \omega)^{\gamma} Q_{\mathrm{S}}}
$$

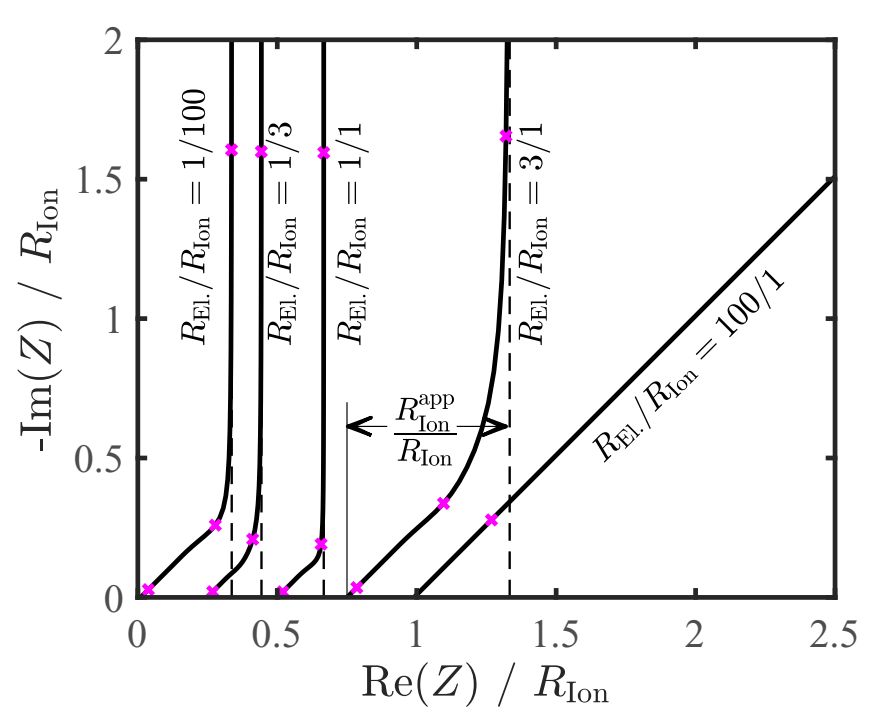

Figure 1. Simulated Nyquist plots $(10 \mathrm{MHz}$ to $1 \mathrm{~Hz})$ of the general transmission line model (Eq. 1) for ratios of electronic to ionic resistances $\left(R_{\mathrm{El}} / R_{\mathrm{Ion}}\right)$ of $1 / 100$ to $100 / 1$ (indicated by the labels in the figure), whereby the axes are normalized to the ionic resistance of the porous electrode $\left(R_{\mathrm{Ion}}\right)$. Here, the absence of charge transfer reactions is assumed $\left(R_{\mathrm{CT}}=\infty\right)$, so that $Z_{\mathrm{S}}$ in Eq. 1 is described by Eq. 6, using an ideal capacitive behavior $(\gamma=1)$ and $Q_{S}=1 \mathrm{mF}$. Magenta crosses mark the frequencies $100 \mathrm{kHz}, 1 \mathrm{kHz}$, and $100 \mathrm{~Hz}$; dashed lines represent the extrapolation of the low-frequency capacitive behavior to the real axis ( $\mathrm{x}$-axis). The difference in $\mathrm{x}$-axis intercepts of the solid lines (modeled impedance response) and the dashed black lines for each resistance ratio corresponds to $1 / 3$ of the apparent ionic resistance $\left(R_{\mathrm{Ion}}^{\mathrm{app}}\right)$.

Eq. 1 reduces to the simplified transmission line model (see Eq. 11 in Ref. 5) only if the electronic resistance $\left(R_{\mathrm{El}} \rightarrow 0\right)$ is insignificant and if no charge transfer reactions are possible $\left(R_{\mathrm{CT}} \rightarrow \infty\right)$. For the simplified transmission line model, plotting the imaginary versus the real impedance in a Nyquist plot, one finds that the difference between the real axis (x-axis) intercept of the $45^{\circ}$ mid-frequency section and between the $\mathrm{x}$-axis intercept of the extrapolated lowfrequency branch is equal to $1 / 3$ of the ionic resistance $\left.\left(R_{\text {Ion }}\right)\right)^{5,8}$ Only under these conditions does the true ionic resistance of the electrolyte within the porous electrode result from the above described Nyquist plot analysis.

On the other hand, if these conditions are not fulfilled, the apparent ionic resistance analogously obtained from the Nyquist plot $\left(R_{\mathrm{Ion}}^{\text {app }}\right)$ is different from the true $R_{\mathrm{Ion}}$-value, and an accurate determination of the tortuosity is only possible if the electronic resistance is known. To evaluate the influence of the electronic resistance contribution $\left(R_{\mathrm{EI}}\right)$ on the apparent ionic resistance $\left(R_{\mathrm{Ion}}^{\mathrm{app}}\right)$ and the error this introduces into the calculated tortuosity values, we simulated Eq. 1 for varying ratios of the ionic to electronic resistances $\left(R_{\mathrm{El}} / R_{\mathrm{Ion}}\right)$. For the sake of simplicity, the absence of charge transfer reactions is assumed (i.e., $R_{\mathrm{CT}}=\infty$ ), which is fulfilled when non-intercalating electrolytes are used. ${ }^{5}$ In this case, the surface impedance elements $Z_{\mathrm{S}}$ of the general transmission line model (Eq. 1) are described by constant phase elements (Eq. 6). For simplicity, we use a constant phase exponent $\gamma=1$ (ideal capacitive behavior) for the following analysis, but the same results would be obtained for other $\gamma$-values.

The simulated Nyquist plots are rescaled in terms of the real ionic resistance and plotted in Figure 1 for ionic to electronic resistance ratios of $100 / 1$ to $1 / 100$. For all ratios, the characteristic transmission line model behavior can be observed, i.e., a distinct $45^{\circ} \mathrm{high} / \mathrm{mid}$ frequency apparent ionic resistance section, followed by the capacitive behavior at low frequencies, with the phase angle approaching $90^{\circ}$ (vertical line on Nyquist plot). For each spectrum, two characteristic resistances can be extracted from the Nyquist plots: a) the apparent high frequency resistance $R_{\mathrm{HFR}}^{\text {app }}$ from the intercept of the modeled high-frequency impedance with the $\mathrm{x}$-axis, and, b) the low-frequency 


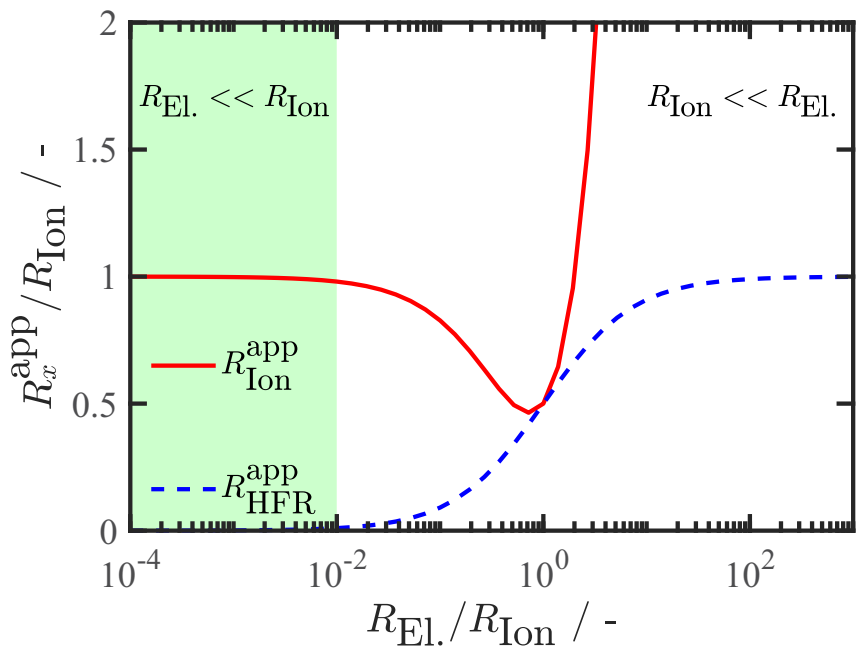

Figure 2. Apparent high frequency resistance (blue line) and apparent ionic resistance (red line) of the simulated Nyquist plots resulting from a general transmission line model (Eq. 1) for various ratios of electronic to ionic resistances $\left(R_{\mathrm{El}} / R_{\mathrm{Ion}}\right)$, normalized to the ionic resistance of the electrolyte in the pores of the electrode. The y-axis values of $R_{x}^{\text {app }} / R_{\text {Ion }}$ represent $R_{\mathrm{HFR}}^{\mathrm{app}} / R_{\mathrm{Ion}}$ in case of the high frequency resistance (blue line) and $R_{\mathrm{Ion}}^{\text {app }} / R_{\mathrm{Ion}}$ in case of the ionic resistance of the electrolyte (red line). Only for $R_{\mathrm{El}}$. $\ll R_{\text {Ion }}$, the apparent ionic resistance and the apparent high frequency resistance approach their real values (indicated by green region).

resistance, represented by the $\mathrm{x}$-axis intercept of the extrapolated low frequency impedance response (indicated by the dashed lines for each spectrum). The difference between the latter and $R_{\mathrm{HFR}}^{\text {app }}$ yields $1 / 3$ of the apparent ionic resistance $R_{\mathrm{Ion}}^{\mathrm{app}}$.

We note that the Nyquist plots shown in Figure 1 are based on a general transmission line model only, which does not include a separate serially connected resistor, which would normally be added to represent electrolyte and cell contacting resistances. Therefore, the change of the apparent high frequency resistance is merely a result of the chosen $R_{\mathrm{El}} / R_{\mathrm{Ion}}$-ratios, while by the conventional definition of the high frequency resistance in battery research, the real high frequency resistance should be zero (i.e., $R_{\mathrm{HFR}}^{\mathrm{app}}=0$ ), as in the here used general transmission line model the separator and cell contacting resistances were omitted and thus effectively set to zero.

As shown by the left-most line in Figure 1, the apparent high frequency resistance approaches its real value of $0 \mathrm{Ohm}$ only when the electronic resistance is small compared to the ionic resistance (i.e., $\left.R_{\mathrm{El}} / R_{\mathrm{Ion}}=1 / 100\right)$. Similarly, only under these conditions the apparent ionic resistance corresponds to the real ionic resistance, indicated by the fact that the X-axis intercept of the $R_{\mathrm{El}} / R_{\mathrm{Ion}}=1 / 100$ line in Figure 1 amounts to $\operatorname{Re}(\mathrm{Z}) / R_{\mathrm{Ion}}=0.33$. The deviation from this idealized case as the ratio of $R_{\mathrm{El}} / R_{\text {Ion }}$ increases, reflecting an increasingly important contribution from electronic resistances in the electrode, is illustrated in Figure 2, plotting the $R_{\text {Ion }}$-normalized apparent resistance vs. $R_{\text {El. }} / R_{\text {Ion }}$ for both the apparent high frequency resistance value and the apparent ionic electrolyte resistance in the electrode pores based on the general transmission line model. As shown at the left side of Figure 2 , for $R_{\mathrm{El}} / R_{\mathrm{Ion}}<10^{-2}$ (green highlighted region), the apparent high frequency resistance (blue dashed line) approaches the real high frequency resistance of $0 \mathrm{Ohm}$ and the apparent ionic resistance approaches its real value (i.e., $R_{\mathrm{Ion}}^{\text {app }} / R_{\mathrm{Ion}}=1$ ). With an increasing $R_{\text {El. }} / R_{\text {Ion }}$ ratio, the apparent high frequency resistance increases (blue line, Figure 2) while the apparent ionic resistance initially decreases (red line). For $R_{\mathrm{El}} / R_{\mathrm{Ion}}=1 / 1$, the total charge at high frequencies is transported effectively through two parallel resistors (ionic and electronic) of the same value, resulting in an apparent high frequency resistance of $R_{\mathrm{HFR}}^{\mathrm{app}}=0.5 \cdot R_{\mathrm{El}}$. (blue line), i.e., $50 \%$ of the electronic resistance of the electrode is added to the measured (apparent) high frequency resistance. At the same time, the apparent ionic resistance decreases to $R_{\mathrm{Ion}}^{\mathrm{app}}=0.5 \cdot R_{\mathrm{Ion}}$ (red line), so that the apparent ionic resistance determined from the Nyquist plot is only half the value of the true ionic resistance. For $R_{\mathrm{El}} / R_{\text {Ion }} \gg 1$, the apparent high frequency resistance approaches the ionic resistance, indicating that essentially all charges are now carried by ionic conduction, while the apparent ionic resistance approaches $1 / 3$ of the electronic resistance (for $R_{\mathrm{El}} / R_{\mathrm{Ion}}=100 / 1$ the $\mathrm{x}$-axis intercept of the low frequency extrapolation yields a value of $\sim 33 \Omega$, not visible in Figure 1 ).

For the determination of the tortuosity $(\tau)$ of porous electrodes, the conductivity of the electrolyte $(\kappa)$, the porosity $(\varepsilon)$ of the electrode (easily determined by electrode thickness $(t)$ and loading measurements) and the effective ionic resistance of the electrolyte within the pores of the electrode $\left(R_{\text {Ion }}\right)$ are required (see Ref. 5).

$$
\tau=\frac{R_{\mathrm{Ion}} \cdot A \cdot \varepsilon \cdot \kappa}{t}
$$

While electrochemical impedance spectroscopy (EIS) is a convenient tool to determine $R_{\text {Ion }}$ via a simplified transmission model, the analysis depicted in Figure 2 shows precise $R_{\text {Ion }}$-values can only be obtained for $R_{\mathrm{El}} / R_{\mathrm{Ion}}<10^{-2}$ (green highlighted region) and that $R_{\text {Ion }}$ would have an error of up to $\sim 20 \%$ for $R_{\mathrm{El}} / R_{\text {Ion }}$ between $10^{-2}$ and $10^{-1}$. For this reason, electrolytes with conductivities as low as possible should be used for the experimental determination of $R_{\text {Ion }}$ via a simplified transmission line analysis. At the same time, higher electrolyte conductivities will lead to a different $R_{\mathrm{Ion}}^{\text {app }} / R_{\mathrm{Ion}}$ value $o n l y$, if the electronic contribution is non-negligible (i.e., if $R_{\mathrm{El}} / R_{\mathrm{Ion}} \gg 10^{-2}$ ). As a consequence, if $R_{\mathrm{Ion}}^{\mathrm{app}} \cdot \kappa$ for identical electrodes remains constant for low (millimolar salt concentrations) and high (molar salt concentrations) electrolyte conductivities, the electronic resistance of the electrode must be negligible, as this condition is only fulfilled in the $R_{\text {El. }} / R_{\text {Ion }}-$ region where $R_{\text {Ion }}^{\text {app }} / R_{\text {Ion }}$ vs. $R_{\text {El. }} / R_{\text {Ion }}$ is constant, i.e., at $R_{\mathrm{El}} / R_{\mathrm{Ion}}<10^{-2}$ (see red line in the green highlighted region in Figure 2$)$. Demonstration of the invariance of $\left(R_{\mathrm{Ion}} \cdot \kappa\right)$ over a wide range of electrolyte conductivities is thus a proof that electronic resistance contributions are negligible and that the extracted $R_{\mathrm{Ion}}^{\text {app }}$-values indeed correspond to the true $R_{\mathrm{Ion}}$-value.

The observant reader might be surprised by the diverging behavior of $R_{\mathrm{Ion}}^{\mathrm{app}} / R_{\mathrm{Ion}}$ for dominating electronic resistances (i.e., for $\left.R_{\mathrm{El}} \gg R_{\mathrm{Ion}}\right)$. If one imagines this limiting case to be corresponding to the model of a separator, a characteristic impedance response of a pure capacitor would be expected (straight vertical line in Figure 1), shifted in the Nyquist plot by the ionic resistance of the separator. Although this might seem to contradict Figure 1 which still shows a $45^{\circ}$ section as well as Figure 2, the capacitive behavior in such a separator cell only stems from the double layer capacitance at the metallic current collector, an interface not taken into account in the transmission line model described above. Further generalization of Eq. 1 allows to include the current collector/coating interface (compare Ref. 7), for which indeed the expected R-C behavior will be observed in the case of a dominating electronic resistance and a negligible double layer capacitance at the surface of the porous material. Because the focus of this work is on the understanding of the influence of the electronic to ionic resistance ratio in a Nyquist plot and for the sake of simplicity, the authors refrain from including interface effects into the above analysis. For the interested reader we note that further generalization will lead to the Nyquist plots in Figure 1, approaching the R-C behavior with the normalized high frequency resistance approaching unity, i.e., the ionic resistance of the porous medium. Correspondingly $R_{\mathrm{Ion}}^{\text {app }} / R_{\mathrm{Ion}}$ in Figure 2 (red line) would not diverge but approach 0 (for $R_{\mathrm{EL}} / R_{\mathrm{Ion}} \gg 1$ ). Up to ionic to electronic resistance ratios of $1 / 1$ the analysis of the transmission line model as conducted above, as well as the generalized version including the contact element to the current collector yield identical results. I.e., in both cases constant $R_{\mathrm{Ion}}^{\mathrm{app}} / R_{\mathrm{Ion}}$ values can only be obtained for negligible electronic resistances (green highlighted region in Figure 2).

In our previous work, we indeed demonstrated the invariance of the measured $R_{\mathrm{Ion}}^{\text {app }}$-values and thus the determined electrode tortuosity for porous graphite electrodes, varying the electrolyte conductivity range 


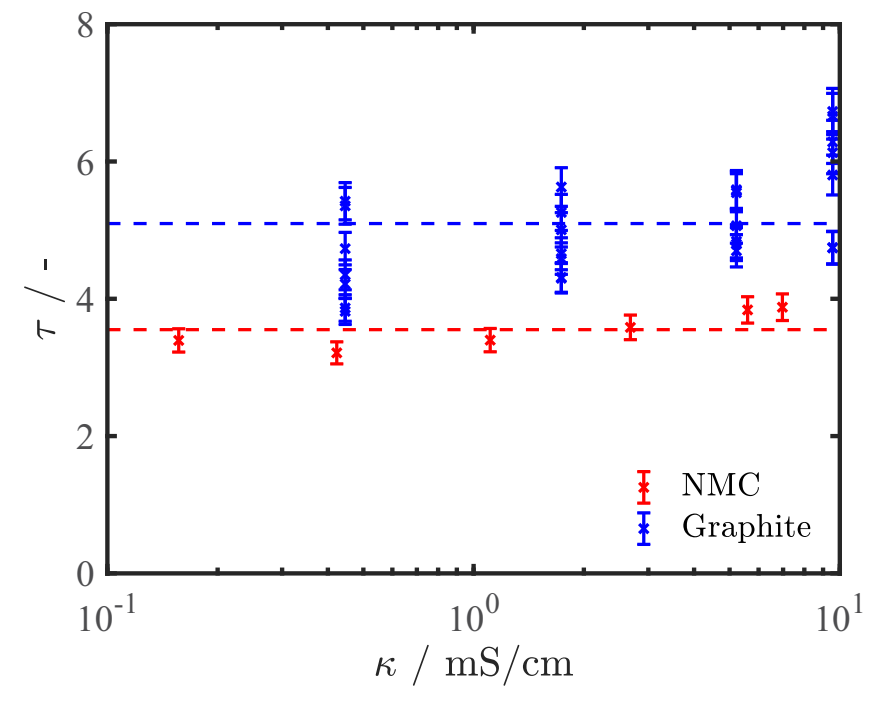

Figure 3. Tortuosity values obtained from simple transmission line model fits of the impedance response of symmetric T-cells using electrolytes with different conductivity. For graphite electrodes (blue symbols; same as in Figure 14 of Ref. 5, with $\varepsilon=43 \%, t=58 \mu \mathrm{m}$, and an areal capacity of $2.1 \mathrm{mAh} / \mathrm{cm}^{2}$ (corresponding to $6 \mathrm{mg} / \mathrm{cm}^{2}$ ), see Ref. 5 for details on electrode preparation and composition), EC:DMC (1:1 w:w) with 10-700 $\mathrm{mM} \mathrm{TBAClO}_{4}$ (conductivities from $0.46-9.56 \mathrm{mS} / \mathrm{cm}$ ) was used. For NMC electrodes (red symbols; from Custom Cells with $\varepsilon=42 \%, t=72 \mu \mathrm{m}, 86 \%$ active material fraction and an areal capacity of $2 \mathrm{mAh} / \mathrm{cm}^{2}$ (corresponding to $13.8 \mathrm{mg} / \mathrm{cm}^{2}$ )), EC:DEC (1:1 w:w) with $\sim 4 \mathrm{mM}-1 \mathrm{M} \mathrm{TBAClO}_{4}$ (conductivities from $0.156-6.97 \mathrm{mS} / \mathrm{cm}$ ). The mean tortuosity values for each electrode are indicated by dashed lines. Error bars indicate a 5\% uncertainty from fitting of the spectra and the conductivity error of the electrolytes.

from $0.45 \mathrm{mS} / \mathrm{cm}$ to $9.6 \mathrm{mS} / \mathrm{cm}$ (re-plotted in Figure 3, blue symbols using the data in Figure 14 of Ref. 5). This was certainly expected, since the electronic conductivity of graphite electrodes is on the order of $>\mathrm{S} / \mathrm{cm},{ }^{9}$ so that $R_{\mathrm{El}} / R_{\text {Ion }}$ quite clearly is $<10^{-2}$, However, for cathode electrodes based on oxide active materials with typically rather low electronic conductivities, e.g., LNMO, ${ }^{10}$ and particularly for cathodes with a low amount of conductive carbon additive, the electronic resistance might not be negligible anymore and the measured value of $\left(R_{\text {Ion }} \cdot \kappa\right)$ could change when switching from low to high conductivity electrolytes. Such a variance of the apparent value of $\left(R_{\text {Ion }} \cdot \kappa\right)$ obtained by impedance analysis when changing the electrolyte conductivity would be a clear indication that the electronic resistance of the electrode cannot be neglected, and that the simple transmission line model would not yield the correct $R_{\text {Ion-value }}$ (i.e., $R_{\mathrm{Ion}}^{\mathrm{app}} \neq R_{\mathrm{Ion}}$ ). To investigate if the electronic contribution becomes significant for electrodes of unknown electronic conductivity, we measured the tortuosity of compressed NMC cathodes (from Custom Cells), using electrolytes based on EC:DEC (1:1 w:w) with different concentrations of $\mathrm{TBAClO}_{4}$ with conductivities ranging from $\left.0.156 \mathrm{mS} / \mathrm{cm}(\sim 4 \mathrm{mM} \mathrm{TBAClO})_{4}\right)$ to $6.97 \mathrm{mS} / \mathrm{cm}\left(\sim 1 \mathrm{M} \mathrm{TBAClO}_{4}\right)$. The obtained tortuosity values, depicted in Figure 3, are proportional to $\left(R_{\mathrm{Ion}}^{\mathrm{app}} \cdot \kappa\right)$ and take slight thickness and porosity variations between the cells into account. Tortuosity values calculated from the measured $\left(R_{\mathrm{Ion}}^{\text {app }} \cdot \kappa\right)$ values for the NMC cathodes (red crosses) and the previously reported graphite anodes ${ }^{5}$ (blue crosses) are essentially constant for electrolyte conductivities varying by a factor of $\sim 45$ $(0.156$ to $6.97 \mathrm{mS} / \mathrm{cm})$. The invariance of the NMC electrode tortuosity values obtained from a simple transmission line model analysis as the electrolyte conductivity is changed over $\sim 1.8$ decades (Figure 3) demonstrates that for both types of electrodes the $R_{\mathrm{El}} / R_{\mathrm{Ion}^{-}}$ ratio must be below $\sim 1 / 100$, i.e., that it must lie within the green region of Figure 2. The resulting tortuosity values of the cathodes with spherical NMC particles are lower $(3.6 \pm 0.3)$ compared to the anisotropic graphite platelets $(5.1 \pm 0.8)$, which is in accord with previous observations. ${ }^{5,11}$

This analysis of the impedance response of porous electrodes over nearly two orders of magnitude in electrolyte conductivity constitutes a rigorous method to validate whether the $R_{\mathrm{Ion}}^{\text {app }}$-values obtained from a simple transmission line model correspond to the true $R_{\text {Ion }}$-values (i.e., whether $R_{\mathrm{El}}$. contributions are indeed negligible). Experimentally, a further reduction of the electrolyte conductivity by reducing the $\mathrm{TBAClO}_{4}$ concentration below $5 \mathrm{mM}$ may lead to increased errors, as small impurities in the cell/porous electrode then could alter the effective ionic conductivity during the measurement. Similarly, the highest conductivity with typical battery electrolyte solvents is limited to $\sim 20 \mathrm{mS} / \mathrm{cm}$. It should be noted that analogous to the experimentally determined $R_{\text {Ion }}^{\text {app }} / R_{\text {Ion }}$ term (here represented in terms of tortuosity), the experimental $R_{\mathrm{HFR}}^{\mathrm{app}}$ values should also be invariant when changing electrolyte conductivity under conditions where $R_{\mathrm{El}}$. can be ignored; an increase of $R_{\mathrm{HFR}}^{\mathrm{app}}$ with increasing electrolyte conductivities would also indicate that the electronic resistance contribution starts to play a role (see blue line in Figure 2). However, changes in $R_{\mathrm{HFR}}^{\mathrm{app}}$ with changing electrolyte conductivities can only be analyzed quantitatively if the cell compression can be controlled precisely (in the case of soft glass fiber separators) or if reasonably incompressible separators are used, so that the high frequency resistance contribution from the separator will be identical for each of the assembled symmetric cells.

In summary, our analysis of the general transmission line model shows that the electronic resistance can influence the apparent high frequency and the apparent ionic resistance obtained from EIS. These theoretical considerations demonstrate that the best condition to obtain the true high frequency resistance and the true ionic resistance is to use an electrolyte with very low conductivity, so that the ionic resistance dominates. Using graphite and NMC electrodes, we showed that if the product of electrolyte bulk conductivity times the apparent ionic resistance $\mathrm{d} R_{\mathrm{Ion}}^{\mathrm{app}} \cdot \kappa\left(\right.$ or $\left.R_{\mathrm{HFR}}^{\mathrm{app}} \cdot \kappa\right)$ is independent over a wide range of bulk electrolyte conductivities (tunable via salt concentration), the electronic resistance contributions are negligible. If the electronic resistance was non-negligible, it could be because the amount of conductive carbon additive is either insufficient or that it is poorly dispersed.

While we have shown above that for negligible electronic resistances in the electrode, the correct ionic resistance within the porous electrode can be extracted from the analysis of the impedance spectra obtained from symmetric cells, it yet remains to be proven that this ionic resistance value can be used to determine the real tortuosity of a porous electrode. Therefore, in the next section, we compare the tortuosity obtained by impedance analysis with the known tortuosity of an arrangement of spherical particles.

\section{Transmission Line Equivalent Circuit Model Validation Using a Macroscopic Setup with Known Tortuosity}

To validate that the tortuosity obtained from the above described EIS method is correct, we study a system with known tortuosity. For a porous medium of spherical particles, the mathematically derived Bruggeman relation allows the calculation of the tortuosity from the porosity of the porous medium ${ }^{12}$

$$
\tau=\varepsilon^{-0.5} \text {. }
$$

In order to apply the EIS method to a packing of spherical particles, a macroscopic symmetric cell setup is built, utilizing stainless chromium steel (type 1.3505) ball bearings of $1 \mathrm{~mm}$ diameter (TIS Wälzkörpertechnologie GmbH, Gauting, Germany) filled into a glass tube to produce a well-defined porous medium of densely packed spheres. As shown in the previous section, a key requirement for the EIS method to be applicable is that the electronic resistance in the porous medium be much smaller than its ionic resistance. For steel ball bearings between two current collectors and compressed using a manual clamp, we find an electronic resistance of $8 \Omega$, which is two orders of magnitude lower than the measured ionic resistance of $790 \Omega$ (see below). 


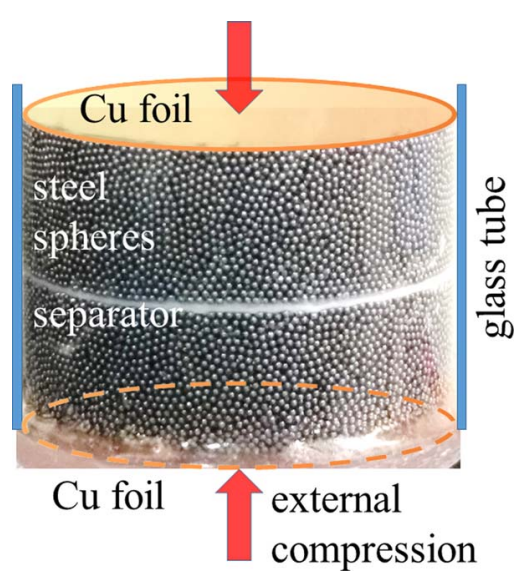

Figure 4. Photograph of the macroscopic symmetric cell setup composed of densely packed spherical steel ball bearings that serves as an idealized porous electrode with known tortuosity. The cell is compressed using a mechanical clamp to ensure a negligible electronic resistance compared to the electrolyte resistance. Experimental parameters of the macroscopic cell setup are given in Table I.

To perform the EIS measurement, the ball bearings are contacted at the top and at the bottom with two copper foil current collectors and are separated in the middle by a stack of glass fiber separators (see Figure 4), creating effectively two stainless steel ball bearing electrodes. The same mechanical pressure is then applied to ensure low electronic resistivity. EC:DMC:DEC with $\sim 30 \mathrm{mM} \mathrm{TBAClO}_{4}$ is used as an electrolyte. The salt concentration is selected such that the ionic conductivity which was determined directly before the experiment, is small compared to the electronic conductivity in the porous medium. Table I lists the specifications for this experiment.

The porosity of the stainless steel ball bearing porous electrodes (SBBPEs) can be obtained from the full geometric volume of the electrodes and either the volume of the electrolyte phase or the volume of the steel ball bearings. With the measured mass of the electrolyte and steel spheres for each of the stainless steel ball bearing electrodes and their respective densities (see Table I), we find porosities of $\varepsilon=38 \%$ and $39 \%$, resulting in tortuosities of $\tau=1.62$ and 1.60 , according to the Bruggeman relation (Eq. 10).

In analogy to the EIS based tortuosity determination for porous lithium ion battery electrodes, ${ }^{5}$ we record an impedance spectrum (Figure 5) and fit it with the simplified transmission line model to extract the ionic resistance of the symmetric cell. An equivalent circuit similar to our previous work was used, i.e., a high frequency resistance from the separator and a serially connected transmission line model for which the electronic resistance was neglected (justified by the electrode compression and the low ionic conductivity, see Table I). From the macroscopic model electrodes composed of densely packed spheres and measured in a symmetric cell configuration, an ionic

\section{Table I. Parameters of the macroscopic symmetric cell setup} depicted in Figure 4.

\begin{tabular}{ll} 
Parameter & Value \\
\hline thickness of one porous electrode & $1.9 \mathrm{~cm}$ \\
radius of the electrode & $2.64 \mathrm{~cm}$ \\
mass of electrolyte per electrode & $16.8 \mathrm{~g}$ \\
mass of steel ball bearings per electrode & $200.4 \mathrm{~g}$ \\
density of electrolyte* & $1.065 \mathrm{~g} / \mathrm{cm}^{3}$ \\
density of steel & $7.9 \mathrm{~g} / \mathrm{cm}^{3}$ \\
electrolyte bulk conductivity at $T_{\text {exp. }}$ & $0.892 \mathrm{mS} / \mathrm{cm}$
\end{tabular}

${ }^{*}$ Measured by weighing $10 \mathrm{ml}$ of electrolyte $\left(\sim 30 \mathrm{mM} \mathrm{TBAClO}{ }_{4}\right.$ in EC:DMC:DEC) directly before the experiment.

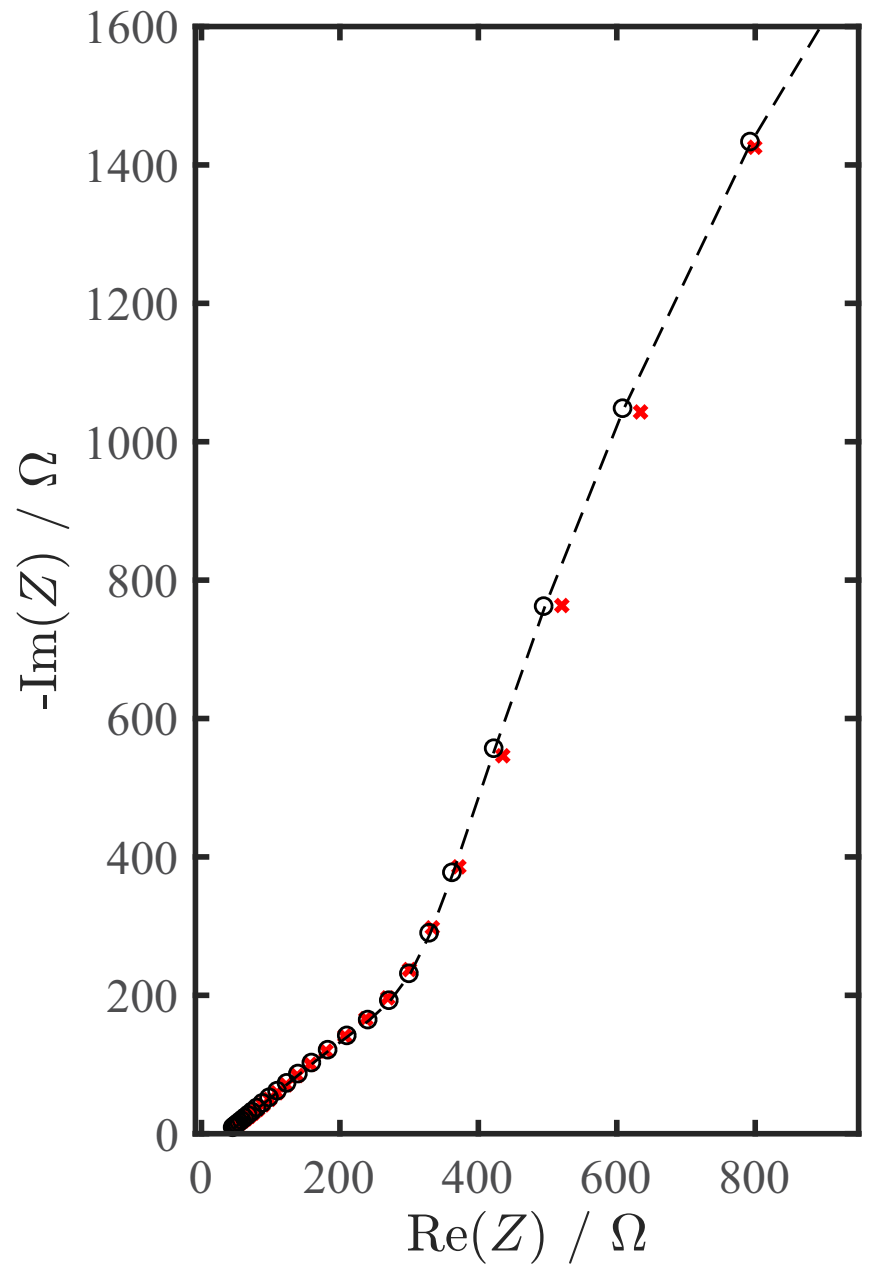

Figure 5. Measured (red crosses) and fitted (black circles) impedance spectrum of the macroscopic model electrodes composed of densely packed spheres shown in Figure 4, using an excitation amplitude of $10 \mathrm{mV}$ in the frequency range from $200 \mathrm{kHz}$ to $20 \mathrm{mHz}$ (the fit and plot range are taken from $1 \mathrm{kHz}$ to 30 $\mathrm{mHz}$ ). The dashed line serves as guide to the eye connecting the measured data points. The fitted values are: $R_{\mathrm{HFR}}=37 \Omega, R_{\mathrm{Ion}}=790 \Omega, R_{\mathrm{CT}}=10200 \Omega$, $Q_{\mathrm{S}}=2.8 \mathrm{mF} \cdot \mathrm{s}^{(\alpha-1)} \Omega$, and $\alpha=0.88$, yielding a tortuosity of 1.56 based on the measured electrode thickness (s. Table I) and an average porosity of $\varepsilon=38.5 \%$.

resistance of $R_{\text {Ion }}=790 \Omega$ for both electrodes is obtained. In this case, a large low-frequency semicircle in the Nyquist plot is observed indicating that the surface impedance elements between the liquid and the solid phase are not described by a constant phase element, but a parallel $R_{\mathrm{CT}} / Q_{\mathrm{S}}$ connection. The large charge transfer resistance $\left(R_{\mathrm{CT}}=10200 \Omega\right)$ may be caused by minor parasitic reactions between the electrolyte and the stainless steel ball bearings. This, however, does not interfere with an accurate determination of the cell's ionic resistance, as is evident from the pronounced $45^{\circ}$ section at high frequencies.

From the ionic resistance $R_{\mathrm{Ion}}=790 \Omega$, the porosity of $\varepsilon=38.5 \pm 0.5 \%$, the thickness of $t=1.9 \mathrm{~cm}$, and a bulk electrolyte conductivity of $\kappa=0.892 \mathrm{mS} / \mathrm{cm}$ (see Table I), we determine a tortuosity of 1.56 for the stainless steel ball bearing electrode. This experimentally determined value is in excellent agreement with the theoretical prediction of $\tau=1.60-1.62$ from the Bruggeman relation.

The $\sim 3 \%$ lower experimental value is likely caused by edge effects in our macroscopic setup: while the Bruggeman relation assumes a perfectly homogeneous porosity throughout the porous medium, the porosity of our SBBPEs is slightly lower close to the surrounding glass cylinder walls where the packing is less dense, which effectively 


\begin{abstract}
Table II. Comparison of tortuosity values $(\tau)$ obtained for the same type of electrodes (Custom Cells) using 3D reconstruction with $\mathrm{X}$-ray tomography (325 $\mathrm{nm}$ voxel size) vs. EIS measurements in a symmetric cell configuration. The uncertainty from the 3D reconstruction is a result of the threshold greyscale value for binarization. Values in brackets indicate the number of repeat measurements for calculation of the EIS-based mean tortuosity value and its standard deviation.
\end{abstract}

\begin{tabular}{lccr} 
Electrode type & Porosity & Coating Thickness & $\tau$ from XTM \\
\hline $\mathrm{NMC}, 3.5 \mathrm{mAh} / \mathrm{cm}^{2} / 24.1 \mathrm{mg} / \mathrm{cm}^{2}, 86 \%$ active material & $40 \%$ & $125 \mu \mathrm{m}$ & $1.77 \pm 0.06$ \\
$\mathrm{NCA}, 3.5 \mathrm{mAh} / \mathrm{cm}^{2} / 21.6 \mathrm{mg} / \mathrm{cm}^{2}, 90 \%$ active material & $40 \%$ & $115 \mu \mathrm{m}$ & $3.1 \pm 0.3(4)$ \\
graphite, $3.8 \mathrm{mAh} / \mathrm{cm}^{2} / 10.9 \mathrm{mg} / \mathrm{cm}^{2}, 96 \%$ active material & $51 \%$ & $110 \mu \mathrm{m}$ & $2.73 \pm 0.03$ \\
\hline
\end{tabular}

reduces the overall measured ionic resistance (and thus the tortuosity) to a small degree.

In summary, the excellent agreement between the theoretically predicted tortuosity and experimentally determined value obtained from the simplified transmission line model equivalent circuit indicates that EIS is a robust method for measuring the tortuosity of porous lithium ion battery electrodes.

\section{Discrepancies Between Tortuosities Obtained from EIS vs. 3D Tomography}

Having demonstrated that neither electronic resistances in the porous electrodes nor the chosen equivalent circuit model introduce errors in the EIS determination of the tortuosity and that there is a good agreement between the tortuosity obtained both theoretically and experimentally for the macroscopic model electrodes, we must understand why the EIS-derived tortuosities are generally higher than the tortuosities obtained by performing numerical diffusion simulations on 3D tomography datasets (compare Figure 21 in Ref. 5).

Here we enable direct comparison between the techniques by analyzing the tortuosity of the same porous electrode using both EIS and numerical diffusion simulations on 3 D reconstructions of the electrode microstructure. For details about the individual techniques, the reader is referred to the original publications (EIS: Ref. 5, X-ray tomography: Refs. 2,11).

Table II summarizes the tortuosities obtained for NMC, NCA, and graphite electrodes (Custom Cells, Itzehoe, Germany). In the case of the numerically calculated tortuosity, the reported values correspond to the value in the through-plane direction (i.e., the direction perpendicular to the separator and the copper foil). In accordance with the general trend in the literature, ${ }^{5}$ even when using the same electrode coatings, a large discrepancy between the tortuosities obtained using both techniques is found (Table II). While XTM-derived tortuosities are in the range of 1.7 to 2.2 for all electrode types, the EIS-derived values are $\sim 2$-fold larger, ranging between 3.1 and 4.0 (the largest discrepancy of a factor $\sim 2.3$ is found for the NCA electrodes).

In the literature it was argued that interactions of the ions with the pore walls, e.g., porous separators, might affect the ionic transport. ${ }^{13}$ It is important to note such interactions (i.e., an interaction between the ions and the surface of the solids) in the electrochemical measurements cannot account for the observed factor of $\sim 2$ between EIS and XTM tortuosities. While generally finite interaction times of the ions may reduce the active ionic concentration in the pores of the coating, somewhere on the order of $50 \%$ of the ions would have to be lost for ionic transport to explain the difference between EIS and XTM tortuosities. However, as evident from Figure 3, stable EIS tortuosity values are observed from $10 \mathrm{mM}$ to $1 \mathrm{M}$ ion concentrations. For geometrical reasons, only a fraction of the ions from a $1 \mathrm{M}$ electrolyte solution can interact with the limited surface area of a porous coating $\left(\sim 100 \mathrm{~cm}^{2} / \mathrm{cm}^{2}\right.$ El. $)$ : e.g., the necessary surface area for half of the ions of a $1 \mathrm{M}$ concentration to be interacting with the pore wall of a $80 \mu \mathrm{m}$ thick coating of $30 \%$ porosity $(1.5 \mu \mathrm{M})$ would be $>2500 \mathrm{~cm}^{2} / \mathrm{cm}^{2}$ El. (conservatively assuming packing of ions without any void space, without solvation shell, and for an ionic radius of 0.3 $\mathrm{nm}$ ). We conclude that any interactions of ionic species with the solid phase in the coating do not significantly alter the EIS tortuosity values (nor the effective ionic conductivity within the pores).
To explore the origin of the lower XTM-derived tortuosity values, it important to consider the origin of errors in the microstructural parameters. First, to measure statistically-relevant, with thousand particles, it is important to measure a volume on the order $1 \mathrm{~mm}^{3}$. With this type of setup, it is not possible to resolve the carbon black and the binder which are generally present in the electrodes, so that only the morphology of the micrometer-scale active material particles can be imaged. The errors caused by the limited resolution $(0.325 \mu \mathrm{m}$ in the examples shown in Table II) have been discussed in the literature, ${ }^{5,14}$ and in general similarly low XTM-derived tortuosities for graphite anodes have been reported (at an even larger voxel size of $0.56 \mu \mathrm{m}){ }^{15}$

High resolution FIB-SEM images actually suggest an inhomogeneous distribution of the conductive carbon/binder phase in the electrode, ${ }^{16}$ making it challenging to assume a distribution. Furthermore, the uncertainty of each tortuosity value derived from XTM stems from the choice of the thresholding greyscale value chosen for binarization of the tomographic information into solid and void space; in the case of EIS-derived tortuosities, the standard deviation of repeat measurements is given (the number of repeat measurements is indicated in Table III parentheses).

When high resolution 3D reconstruction techniques are used, higher tortuosities are found, e.g., values ranging from 2 to 7 are reported for MCMB (mesocarbon microbeads) anodes, but in this case the reconstructed volumes $(15 \mu \mathrm{m}$ sized cubes) are likely far too small to be representative for the entire electrode, due to the fact that the spherical MCMB particles already have a diameter of $\sim 8 \mu \mathrm{m} .{ }^{17}$ Considering these difficulties, it is not surprising that $3 \mathrm{D}$ reconstructions of representative coating volumes only yield higher tortuosities, more in agreement with the electrochemical results, if the binder/carbon matrix is added computationally to the active material reconstruction. ${ }^{18}$ An entirely different experimental approach is based on measuring the permeation rate of gas along the in-plane direction of an electrode (parallel to the current collector) using a flow-field setup, which also confirms the generally higher tortuosity values obtained by EIS. For example, a tortuosity of $\sim 6$ for a graphite electrode with $30 \%$ porosity (50 $\mu \mathrm{m}$ thickness) is reported, ${ }^{14}$ which is in good agreement with the EIS-derived tortuosity value of $\sim 5.5$ which have been obtained for similarly compressed graphite electrodes. ${ }^{5}$

We conclude that the binder and carbon black phases are the most likely reason for the underestimation of the tortuosity of porous electrodes. To validate this hypothesis, we prepared anode coatings with graphite (T311, SGL Carbon, $3.0 \mathrm{~m}^{2} / \mathrm{g}$ BET, D50 diameter of $19 \mu \mathrm{m}$ ) with different weight percentages of PVDF binder (Kureha KF 1100) in order to determine their tortuosity by EIS. We deliberately chose extreme binder weight percentages of $1.5 \%$ (minimum content to prepare stable electrodes) and $10 \%$ (highest reasonable content).

Figure 6 shows the Nyquist plots from symmetric T-cells $\left(0.95 \mathrm{~cm}^{2}\right.$ area) with $10 \mathrm{mM} \mathrm{TBAClO}_{4}$ in EC:DEC $(\kappa=0.423 \mathrm{mS} / \mathrm{cm})$ for uncompressed graphite electrodes with $1.5 \%$ PVDF binder (blue; $109 \pm 2 \mu \mathrm{m}$ coating thickness, $51 \pm 0.5 \%$ porosity) and with $10 \%$ PVDF (red; $85 \pm 2 \mu \mathrm{m}$ coating thickness, $50 \pm 0.5 \%$ porosity). Two measurements are conducted for each electrode type, and the impedance response is shifted by the high frequency resistance to facilitate comparison. From the given experimental parameters (porosity, thickness, electrolyte conductivity, and area) and the EIS-derived ionic resistances of $R_{\text {Ion }}=145 \pm 5 \Omega$ and $213 \pm 4 \Omega$, tortuosities of $2.7 \pm 0.1$ and $5.0 \pm 0.2$ are obtained for electrodes with $1.5 \%$ and 


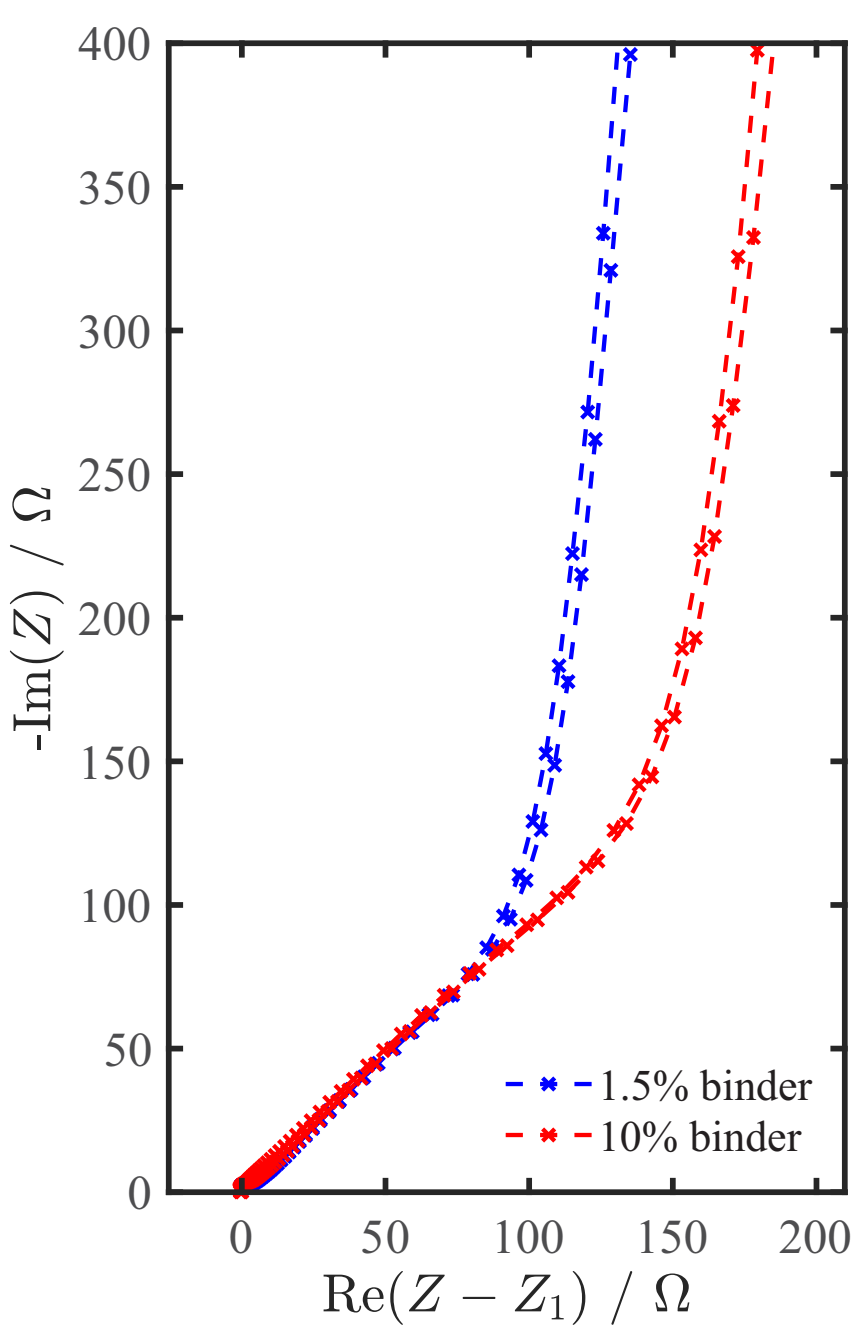

Figure 6. Nyquist plots of nominally identical symmetric T-cells $\left(0.95 \mathrm{~cm}^{2}\right.$ area) with uncompressed graphite (T311, SGL Carbon, $3.0 \mathrm{~m}^{2} / \mathrm{g}$ BET, D50 diameter of $19 \mu \mathrm{m}$ ) electrodes with $1.5 \%$ (blue) and 10\% (red) PVDF binder (Kureha KF 1100). Measurements were conducted with $10 \mathrm{mM} \mathrm{TBAClO}{ }_{4}$ in EC:DEC $(\kappa=0.423 \mathrm{mS} / \mathrm{cm})$, using an excitation amplitude of $20 \mathrm{mV}$ in the frequency range from $200 \mathrm{kHz}$ to $100 \mathrm{mHz}$. The spectra were shifted along the $\mathrm{x}$-axis to the origin for easier comparability.

$10 \%$ binder respectively. Thus, even though the binder is a small fraction of the coating mass and volume $(1 \%-5 \%)$, it has a pronounced effect on the effective ionic transport properties of the porous electrode and could explain the difference between the tortuosity measured by numerical diffusion of the $3 \mathrm{D}$ reconstructions and that measured by EIS.

In summary, this work highlights that tortuosity measured by numerical diffusion of the 3D reconstructions will only be as accurate as the $3 \mathrm{D}$ reconstructions themselves, i.e., for a large representative volume and if a high contrast allows to discriminate the binder/carbon matrix from the electrode void volume. Further experimental investigations about the influence of the binder on the electrochemically determined tortuosity for varying electrode compositions have been conducted and will soon be published in a detailed, separate study.

\section{Conclusions}

Previously reported tortuosities, determined by electrochemical impedance spectroscopy (EIS) measurements using a symmetrical cell configuration and a non-intercalating electrolyte, were based on the assumptions that the electronic resistance of the electrode is negli- gible and that ionic transport in the electrolyte phase within the pores of an electrode is represented correctly by a transmission line model. Under these conditions, the electrode tortuosity can be determined from the ionic resistance extracted from the impedance spectrum. In the present work, we validated both assumptions based on theoretical considerations and experiments. Thus, we demonstrated an experimental methodology by which the assumption of negligible electronic resistance contributions to the electrode impedance measured in symmetrical cells with a non-intercalating electrolyte can be verified: the assumption is fulfilled, if the product of the measured ionic resistance times the electrolyte bulk conductivity is independent of the bulk electrolyte conductivity, which can be adjusted by the salt concentration. This was illustrated for typical graphite anodes and NMC cathodes, both of which showed negligible electronic resistance contributions, allowing for the quantification of their tortuosity.

The validity of the transmission line model analysis was furthermore examined for a macroscopic representation of a porous electrode constructed from electronically conducting steel spheres which were densely packed into an electrolyte filled glass cylinder and separated mid-way by a porous separator. The impedance-derived tortuosity value was shown to be essentially identical with the value predicted by the Bruggeman relation, which was derived for this geometry. This unequivocally proves that the EIS-derived tortuosity values are indeed correct, as long as electronic resistance contributions can be neglected, whereby the latter assumption can be examined in a straightforward fashion (see above).

After having validated the accuracy of the EIS method, we investigated the reason for the $\sim 2$-fold lower tortuosities derived from $3 \mathrm{D}$ reconstructions based on X-ray tomography compared to EIS-derived values, using identical electrodes cut from the same coating. From electrodes with deliberately chosen low and high binder contents, we conclude that the (at least partially) unresolved binder and conductive carbon phases due to the insufficient resolution of 3D reconstructions is the most likely cause for the pronounced underestimation of electrode tortuosities.

\section{Acknowledgment}

J. L. gratefully acknowledges the funding by the BMBF (Federal Ministry of Education and Research, Germany) for its financial support under the auspices of the ExZellTUM II project (grant number 03XP0081). M. E. and V. W. acknowledge the ERC (grant number 680070). Special thanks go to Andreas Ehrl for helpful discussions and Patrick Pietsch for help with X-ray tomography.

\section{ORCID}

Johannes Landesfeind (1D https://orcid.org/0000-0003-0333-2185 Vanessa Wood (1D https://orcid.org/0000-0001-6435-0227

\section{References}

1. X. G. Yang, Y. Leng, G. Zhang, S. Ge, and C. Y. Wang, J. Power Sources, 360, 28 (2017).

2. M. Ebner, F. Geldmacher, F. Marone, M. Stampanoni, and V. Wood, Adv. Energy Mater, 3, 845 (2013).

3. J. Joos, T. Carraro, A. Weber, and E. Ivers-Tiffée, J. Power Sources, 196, 7302 (2011)

4. N. Ogihara, S. Kawauchi, C. Okuda, Y. Itou, Y. Takeuchi, and Y. Ukyo, J. Electrochem. Soc., 159, A1034 (2012).

5. J. Landesfeind, J. Hattendorff, A. Ehrl, W. A. Wall, and H. A. Gasteiger, J. Electrochem. Soc., 163, A1373 (2016).

6. D. Cericola and M. E. Spahr, Electrochim. Acta, 191, 558 (2016).

7. H. Göhr, in Electrochemical Applications, p. 2, ZAHNER-elektrik GmbH \& Co. KG (1997).

8. Andrzej Lasia, Electrochemical Impedance Spectroscopy and its Applications, Springer, (2014)

9. M. Park, X. Zhang, M. Chung, G. B. Less, and A. M. Sastry, J. Power Sources, 195 , 7904 (2010).

10. H. Liu, J. Wang, X. Zhang, D. Zhou, X. Qi, B. Qiu, J. Fang, R. Kloepsch, G. Schumacher, Z. Liu, and J. Li, ACS Appl. Mater. Interfaces, 8, 4661 (2016). 
11. M. Ebner, D. W. Chung, R. E. García, and V. Wood, Adv. Energy Mater, 4, 1 (2014).

12. D. A. G. Bruggeman, Ann. Phys., 416, 636 (1935).

13. M. F. Lagadec, M. Ebner, R. Zahn, and V. Wood, J. Electrochem. Soc., 163, A992 (2016).

14. T. DuBeshter, P. K. Sinha, A. Sakars, G. W. Fly, and J. Jorne, J. Electrochem. Soc., 161, A599 (2014)
15. C. Lim, B. Yan, L. Yin, and L. Zhu, Energies, 7, 2558 (2014).

16. L. Zielke, T. Hutzenlaub, D. R. Wheeler, C. Chao, I. Manke, A. Hilger, N. Paust, R. Zengerle, and S. Thiele, Adv. Energy Mater., 1 (2014).

17. F. Tariq, V. Yufit, M. Kishimoto, P. R. Shearing, S. Menkin, D. Golodnitsky, J. Gelb, E. Peled, and N. P. Brandon, J. Power Sources, 248, 1014 (2014).

18. L. Zielke, T. Hutzenlaub, D. R. Wheeler, I. Manke, T. Arlt, N. Paust, R. Zengerle, and S. Thiele, Adv. Energy Mater., 4, 1 (2014). 